\title{
DESARROLLO SOSTENIBLE E INSTRUMENTOS ECONÓMICOS AMBIENTALES: UNA DISCUSIÓN PARA AMÉRICA LATINA Y EL CARIBE
}

\author{
SUSTAINABLE DEVELOPMENT AND ENVIRONMENTAL ECONOMIC \\ INSTRUMENTS: A DEBATE FOR LATIN AMERICA AND THE CARIBBEAN
}

\author{
MAURICIO PEREIRA* \\ Comisión Económica para América Latina y el Caribe, Chile \\ mauricio.pereira@cepal.org
}

\section{INTRODUCCIÓN}

El concepto de desarrollo sostenible se entiende como un estilo de desarrollo que integra en forma equilibrada tres dimensiones fundamentales del entorno de las personas: el económico, el social, y el ambiental. Este estilo de desarrollo permitiría lograr que "se satisfagan las necesidades del presente sin comprometer la capacidad de las generaciones futuras para satisfacer sus propias necesidades" (WCED 1987).

Este concepto se masifica, recién en el año 1987, a partir del reporte Our Common Future elaborado por la comisión Brundtland. En este documento se analizaba como los patrones de crecimiento económico se habían relacionado con un uso irracional de recursos naturales, altos niveles de pobreza e impactos ambientales. También se señalaba que para revertir estas tendencias preocupantes, previstas por el Club de Roma y plasmadas en el documento Limits of Growth (Meadows et. al. 1972), era necesario un cambio de paradigma que se centrara en un desarrollo sostenible.

Históricamente, el crecimiento económico en América Latina y el Caribe (ALC) ha presentado una deficiente alineación con los patrones de desarrollo social y un escaso cuidado medio ambiental (Prebisch 1980). Este hecho empeoró en la década de 1980 en la llamada década pérdida, aunque posteriormente ha existido una tendencia a la recuperación de patrones más sostenibles (Ocampo y Ros 2011).

En la región es posible diferenciar dos patrones de especialización productiva. En primer lugar, aparece el de América del Sur, que se enfoca en la explotación de

Las opiniones expresadas en este documento son de exclusiva responsabilidad del autor y pueden no coincidir con las de la organización a la que pertenece.

Revista de Gestión Pública

VOLUMEN I, Número 1

ISSN 0719-1820

pp. $165-175$ 
los recursos naturales. El avance de este patrón, en forma no sostenible, generaría el agotamiento de recursos (forestales y pesqueros), pérdidas de capital natural, contaminación al aire, agua y suelos (Ocampo y Ros 2011). En segundo lugar, se aprecia el patrón de México, Centroamérica y el Caribe, que se caracteriza por la industria de la maquila ${ }^{1}$ y del turismo. Este patrón podría llevar consigo problemas de hacinamiento cuando no ha sido planificada la ubicación de los trabajadores. Mientras que el turismo no sostenible puede generar contaminación del borde costero y debilitar los ecosistemas marinos y terrestres que se desarrollan en ese entorno.

Tanto el crecimiento poblacional de la región, que en los últimos treinta años ha aumentado en doscientos setenta millones de personas, como los cambios en los hábitos de consumo y la necesidad de mayores y mejores servicios han ejercido enormes presiones sobre el medio ambiente y los recursos naturales (Tabla 1).

La frontera agrícola se ha expandido en casi treinta millones de hectáreas, lo que junto con el crecimiento horizontal de los suelos urbanos ha ido en desmedro de la superficie boscosa, que en sólo veinte años se ha reducido en noventa y tres millones de hectáreas, causando importantes pérdidas en los bosques nativos y en la diversidad biológica de la región.

La extracción pesquera ha sido excesiva llegando a la sobre explotación y al agotamiento de muchas especies (FAO 2007, PNUMA 2011). La extracción de minerales se ha incrementado notablemente, incluso tres veces más que el crecimiento de la población regional. Otros incrementos importantes se aprecian en la extracción de petróleo, en el consumo energético y en las emisiones de CO2.

Manufacturas de bajo y medio nivel tecnológico con uso intensivo de mano de obra barata 
Debates y Reseñas

Tabla 1: Indicadores físicos para América Latina y el Caribe: 1990-2010

\begin{tabular}{|c|c|c|c|c|c|c|}
\hline & 1990 & 1995 & 200 & 2005 & 2010 & $\begin{array}{c}\text { Variación } \\
1990-2010\end{array}$ \\
\hline $\begin{array}{c}\text { Población } \\
\text { (Miles de personas) }\end{array}$ & 443.032 & 482.647 & 521.429 & 557.038 & 590.082 & $33 \%$ \\
\hline $\begin{array}{c}\text { Densidad } \\
\text { poblacional } \\
\text { (Personas por Km2) }\end{array}$ & 21,7 & 23,6 & 25,6 & 27,3 & 29,0 & $34 \%$ \\
\hline $\begin{array}{c}\text { Superficie cubierta } \\
\text { por bosques } \\
\text { (Miles de hectáreas.) }\end{array}$ & 1.039 .686 & --- & 990.707 & 966.504 & 946.756 & $-9 \%$ \\
\hline $\begin{array}{c}\text { Superficie Agrícola } \\
\text { (Miles de hectáreas) }\end{array}$ & 683.736 & 703.963 & 710.321 & 711.737 & $712.065^{\mathrm{b}}$ & $4 \%$ \\
\hline $\begin{array}{c}\text { Captura pesquera } \\
\text { (Toneladas) }\end{array}$ & 16.161 .861 & 21.583 .811 & 19.987 .121 & 18.758 .194 & $16.216 .696^{\mathrm{b}}$ & $0.3 \%$ \\
\hline $\begin{array}{c}\text { Producción agrícola } \\
\text { (Miles de toneladas) }\end{array}$ & 5.367 & 5.970 & 5.921 & 5.735 & $6.248^{\mathrm{a}}$ & $16 \%$ \\
\hline $\begin{array}{c}\text { Extracción mineral } \\
\text { (Miles de toneladas) }\end{array}$ & 262.382 & 310.189 & 345.909 & 526.819 & $513.174^{\mathrm{b}}$ & $96 \%$ \\
\hline $\begin{array}{c}\text { Extracción de } \\
\text { petróleo }\end{array}$ & 1.006 .215 & 1.153 .017 & 1.321 .016 & 1.450 .691 & $1.637 .385^{\mathrm{b}}$ & $63 \%$ \\
\hline $\begin{array}{c}\text { (Miles de toneladas) } \\
\text { Carbono emitido } \\
\text { (Miles de toneladas } \\
\text { de CO2) }\end{array}$ & 341.422 & 405.146 & 474.207 & 514.762 & $489.606^{\mathrm{b}}$ & $43 \%$ \\
\hline $\begin{array}{c}\text { Consumo energético } \\
\text { (Miles de baúles equi- } \\
\text { valentes en petróleo) }\end{array}$ & 1.718 .146 & 1.982 .851 & 2.300 .450 & 2.529 .872 & $2.822 .383^{\mathrm{c}}$ & $64 \%$ \\
\hline
\end{tabular}

a: 2007, b: 2008, c: 2009

Fuente: Elaboración propia sobre la base de CEPALSTAT y FAOSTAT.

En términos económicos, el modelo de crecimiento de la región se ha caracterizado por el uso indiscriminado de recursos naturales, una limitada diversificación productiva, una baja inversión en innovación y tecnología. Este hecho se observa en el siguiente gráfico, donde el 2008 más del 65\% de las exportaciones de la región eran primarias o se basaban en recursos naturales y solo un $12 \%$ de las exportaciones eran intensivas en alta tecnología ${ }^{2}$.

\footnotetext{
2 Sin México, las exportaciones de ALC serían en un 71\% productos primarios o se basados en recursos naturales y solo un $4 \%$ serían productos de alta tecnología.
} 


\section{Gráfico 1: América Latina y el Caribe: Composición del comercio de bienes}

2008
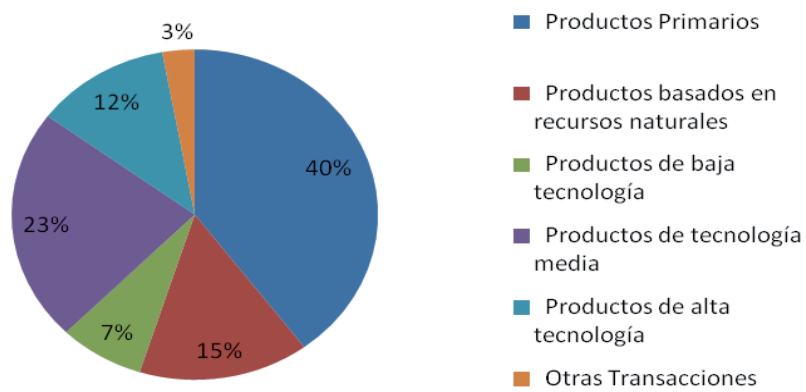

Fuente: Elaboración propia sobre la base de CEPALSTAT.

Lo anterior ha ocasionado que los recursos que se generan en la región no se dispersen a través de encadenamientos productivos y que no se creen tejidos industriales más extensos. No obstante, la región ha aumentado su nivel de ingresos anualmente en más de un 3\% en promedio durante las últimas dos décadas y a fines del 2010 el ingreso per cápita superaba los 5.000 dólares $^{3}$. Sin embargo, existe una amplia heterogeneidad entre los países de la región como el caso de Bahamas que bordea los 17.000 dólares per cápita y el de Haití que apenas alcanza los 360 dólares.

Por otro lado, la brecha de ingresos ha aumentado en relación con los países más desarrollados lo que muestra una nula convergencia hacia las economías más adelantadas. Por ejemplo, si comparamos el PIB per cápita ajustado por poder adquisitivo de ALC con el de Estados Unidos, la Unión Europea y la OCDE (Gráfico 2 a) se aprecia que el diferencial de ingresos ha ido en permanente aumento en el tiempo. Mientras que si lo comparamos con el ingreso promedio mundial no se aprecia una ganancia relativa.

A su vez, si comparamos el diferencial de ingresos normalizados a 1980 para 10 países de ALC (Gráfico 2b) con respecto a Estados Unidos, se aprecia que la brecha se ha incrementado en el tiempo para todos los países aunque en términos diferenciados. Por ejemplo, el país que menos ha incrementado su brecha con respecto a Estados Unidos es Chile, mientras el que más ha aumentado su brecha es Venezuela. 
Gráfico 2: Brecha de Ingreso per cápita (dólares de 2005 PPP)

a) Diferencial de ingreso de América Latina y el Caribe con respecto a países más desarrollados

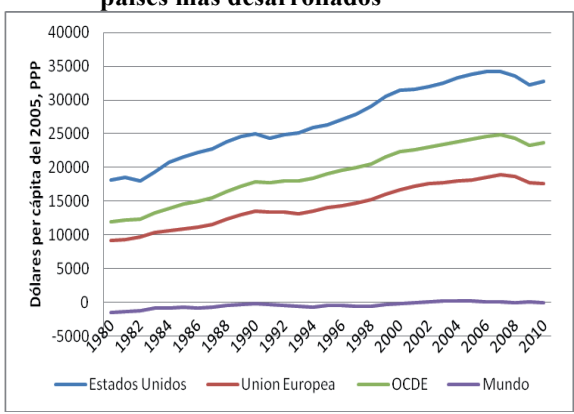

b) Diferencial de ingreso normalizado de países con respecto a USA

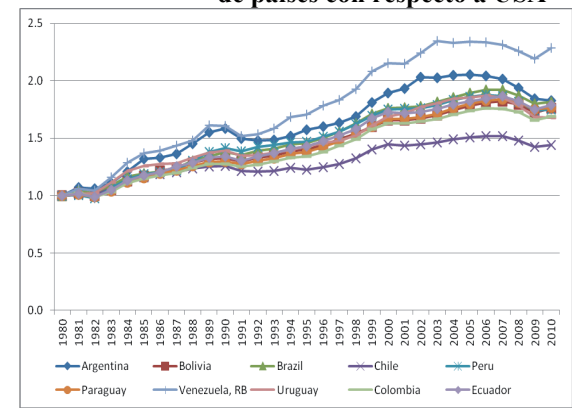

Fuente: Elaboración propia sobre la base de World Development Indicators.

En términos sociales, ALC aún presenta una alta tasa de pobreza, aunque ésta ha disminuido fuertemente en los últimos años desde casi la mitad de la población de la región en 1990 hasta llegar a un tercio de ella en 2010. Lamentablemente, aún 180 millones de personas se encuentran en esta condición. Al mismo tiempo, ALC todavía se encuentra como la región más desigual del mundo, hecho que se manifiesta en el índice de Gini, lo que muestra una gran concentración de la riqueza y presenta enormes desafíos por lograr una distribución más equitativa. En este sentido surge con fuerza el rol del Estado el que en promedio para la región todavía presenta un bajo aporte social, con un gasto en educación y en salud de solo el 4\% del PIB para cada uno, cifras muy lejanas a las de la OECD que aporta un $5.4 \%$ y un $7.5 \%$ del PIB respectivamente.

La cobertura educacional se mantiene baja, si bien se han hecho importantes avances en la cobertura universal de educación primaria, solo muy pocos países de la región cumplirán con la meta de desarrollo del milenio asociada para el 2015 y la cobertura secundaria aún se mantiene muy rezagada (IADB 2005, CEPAL 2010a). Por otro lado, la calidad de la educación sigue siendo deficiente, como lo muestra la prueba PISA del año 2009. Esto se materializa en los resultados para Chile, Uruguay y México, los que pese a encontrarse dentro de los países mejores indicadores de la región, solo ubica a estos países dentro de los 40 con mejores indicadores dentro de los 62 encuestados. A su vez, los indicadores y metas en salud siguen mostrándose deficitarios.

Uno de los principales indicadores que sintetizan el desarrollo sostenible de un país es el ahorro genuino (AG). Este indicador se construye bajo el concepto de cuentas nacionales ambientales y mide la "verdadera" tasa de ahorro de una economía al tomar en cuenta la inversión en capital humano, el agotamiento de los recursos naturales y el daño causado por las emisiones. Tal como se muestra en la 


\section{Debates y Reseñas}

Tabla 2 el ahorro genuino de la región ha ido cayendo en el tiempo y actualmente se mantienen en torno al 7\% del PIB mientras que el ahorro bruto estaría en torno al 19\%. Esto implica que en promedio la región ha perdido capital físico, natural y social en torno al 12\% del PIB dejando un 7\% del PIB de excedentes. Un tema a tener en cuenta es el hecho que existen países en la región que presentan un ahorro genuino negativo (pese a tener un ahorro bruto positivo), lo que implica una pérdida de bienestar inter generacional en la sociedad.

Otro indicador que entrega una idea de cuán lejos se encuentra una sociedad de la meta ideal de desarrollo es el Índice de Desarrollo Humano (IDH) ya que considera tanto la magnitud del crecimiento económico como la calidad del mismo, y en este sentido también toma en cuenta la desigualdad. En términos prácticos, el IDH se mide a través de en tres dimensiones: salud, educación e ingresos. El IDH regional se encuentra actualmente en 0.704 presentando un desarrollo alto a nivel mundial aunque al compararnos con la OECD (0.879) nos muestra aún una brecha por reducir. Sin embargo, si este indicador se ajusta por desigualdad nuestra región pierde importantes posiciones y supera únicamente al Sudeste Asiático y a África Sub-Sahariana.

Tabla 2: Principales indicadores para América Latina y el Caribe: 1990-2010

\begin{tabular}{|c|c|c|c|c|c|c|}
\hline & & 1990 & 1995 & 1995 & 2005 & 2010 \\
\hline Ahorro bruto & $\%$ del PNB & 19.8 & 17.5 & 17.9 & 22.0 & 19.1 \\
\hline $\begin{array}{l}\text { Ahorro genuino, incluyen- } \\
\text { do daño por emisiones }\end{array}$ & $\%$ del PNB & 7.8 & 7.7 & 6.2 & 7.9 & 6.8 \\
\hline PIB per cápita (CEPAL) & $\begin{array}{l}\text { Dólares constan- } \\
\text { tes del } 2000\end{array}$ & 3554 & $\begin{array}{c}3 \\
809\end{array}$ & 4124 & 4389 & 5040 \\
\hline PIB per cápita (BM) & $\begin{array}{l}\text { Dólares constan- } \\
\text { tes del } 2005 \text { PPP }\end{array}$ & 6969 & $\begin{array}{c}7 \\
554\end{array}$ & 8126 & 8665 & 9918 \\
\hline Tasa de Pobreza ${ }^{a}$ & \% de la población & 48.3 & 45.7 & 43.9 & 39.8 & 33.1 \\
\hline $\begin{array}{l}\text { Coeficiente de Gini } \\
(\mathrm{x} 100)\end{array}$ & $\begin{array}{l}\text { Valor entre } \\
0-100\end{array}$ & 54 & -- & 55 & -- & 52 \\
\hline Gasto en Salud $^{a}$ & $\%$ del PIB & -- & 3.1 & 3.2 & 3.3 & 3.9 \\
\hline Gasto en Educación ${ }^{a}$ & $\%$ del PIB & -- & 4.0 & 4.2 & 3.9 & 3.9 \\
\hline $\begin{array}{c}\text { Índice de Desarrollo } \\
\text { Humano (IDH) }\end{array}$ & a: Correspolnde a A & épiel & tinā- & 0.660 & 0.681 & 0.704 \\
\hline
\end{tabular}

Fuente: Elaboración propia sobre la base de Banco Mundial, CEPAL y PNUD. 


\section{LOS DESAFÍOS POR VENIR}

El modelo de desarrollo imperante en la región, pese a causar un agotamiento del medio y un alto nivel de desigualdad, ha logrado en cierta medida aumentar los ingresos y reducir la pobreza en general. Sin embargo, estos avances se encuentran en peligro por diversos factores externos que podrían afectar en distinto grado a cada país de ALC, dependiendo de las medidas que se tomen.

La crisis financiera que comenzó a fines del 2008 ha generado que la mayoría de las economías desarrolladas (Estados Unidos, Japón y la Unión Europea) disminuyan en forma notable su crecimiento económico. Este hecho ha generado que contraigan su demanda por materias primas, afectando directamente a las exportaciones de ALC. Sin embargo, y debido al fuerte impulso económico que ha tenido China y Asia en general, la crisis financiera que comenzó el 2008 no se ha hecho sentir en ALC con la magnitud que se esperaría. Los elevados precios de los minerales y de los alimentos han permitido que las exportaciones operen como un motor de crecimiento de la región.

En este sentido y de acuerdo con CEPAL (2011) se espera que el valor del comercio de la región crezca un $27 \%$ en el 2011 , el que se descompone en un aumento vía precios del $18 \%$ y de volumen del $9 \%$. Luego, cualquier volatilidad en los precios internacionales afectaría directamente el valor de las exportaciones en la región. En este sentido es necesario recordar la importancia de los fondos de estabilización que ahorran en el extranjero los ingresos obtenidos de los recursos naturales y que pueden ser gastados en circunstancias especiales. Resalta el fondo de estabilización del petróleo de Noruega y el del cobre en Chile como mecanismo estabilizador de precios e impactos externos ${ }^{4}$.

Previo a la crisis financiera la preocupación mundial se asociaba con el fuerte incremento de los alimentos. En este sentido se espera que una vez que las economías desarrolladas recuperen su crecimiento vuelva a surgir con fuerza este tema. Si no se toman medidas preventivas ahora, es posible que en un futuro próximo el aumento del precio de los alimentos, generados por aumentos en los costos de los fertilizantes y el transporte y los cambios en los hábitos de consumo de los países emergentes, pueda generar que los grupos con menores ingresos y más desprotegidos de nuestra sociedad vean comprometidos sus niveles mínimos de consumo por subsistencia.

\footnotetext{
La idea de los fondos de estabilización va de la mano con concepto de sostenibilidad débil de Hartwick, el que señala que es necesario mantener en el tiempo un stock de capital constante el que se puede logar al sustituir el capital natural exportado por otros tipos de capital (financiero, educacional o físico, entre otros).
} 
Otro factor que podría generar importantes transformaciones en nuestra sociedad es el cambio climático. Diversos autores (CEPAL 2010b, Sachs 2008, Stern 2007) argumentan que los cambios en los patrones de temperatura y precipitación del clima generarán múltiples efectos de carácter multidimensional. De hecho, se esperarían importantes alteraciones en la productividad de los cultivos agrícolas lo que obligaría a desplazar cultivos y aplicar medidas de adaptación, se pronostica un aumento del nivel medio del mar en torno a un metro lo que afectaría zonas turísticas puertos y asentamientos humanos. Las variaciones en precipitaciones afectarían los recursos hídricos y sus causes. Los cambios en temperatura y humedad incrementarían y expandirían las enfermedades causadas por vectores como la malaria y el dengue. Los eventos extremos aumentarían su frecuencia e intensidad. En suma, estos impactos necesitarán la acción del Estado para brindar y proveer tecnología y fondos para la adaptación y la mitigación de este fenómeno.

\section{INSTRUMENTOS AMBIENTALES Y CAMBIOS DE PARADIGMAS}

ALC se encuentra ante la oportunidad de realizar los cambios necesarios para modificar sus patrones de crecimiento y hacerlos más sostenibles y equitativos. En este punto, surge con fuerza la importancia y el rol activo del Estado como un agente encargado de coordinar, entregar los estímulos necesarios con el fin de orientar las políticas públicas.

Según CEPAL (2010c) el rol del Estado debería normar cinco áreas específicas con el fin de mejorar la situación de los pueblos de ALC en el futuro. A continuación, se señalan estos puntos:

1. Garantizar un entorno macroeconómico adecuado para prevenir y enfrentar la volatilidad y robustecer las economías mediante el uso de los instrumentos disponibles (financieros, fiscales y cambiarios) y así promover un patrón de crecimiento hacia un mayor dinamismo productivo con empleo de calidad.

2. Promover cambios en la matriz productiva, propiciando una mayor incorporación de tecnologías, una menor intensidad de carbono e impulsar políticas destinadas a reducir las brechas de productividad entre los distintos sectores y niveles de la estructura productiva.

3. Reducir las disparidades territoriales, tanto en el ámbito de las capacidades productivas, la articulación con mercados ampliados, la convergencia y el acceso a servicios como en cuanto a los niveles de bienestar. Revertir la situación de desigualdad territorial implica atacar la desigualdad general.

4. Impulsar políticas activas y pasivas en el ámbito del empleo y del cuidado, a fin de prevenir el desempleo, cerrar las brechas de ingresos laborales 
como mecanismo clave de promoción de la igualdad e incrementar las tasas de participación y empleo.

5. Garantizar un mayor acceso al bienestar y un mayor desarrollo de las capacidades de los sectores más rezagados y vulnerables, lo que contribuye sustancialmente a cerrar las brechas sociales. En materia social, el rol del Estado es clave en los pilares de protección y promoción. Se propone diseñar y establecer un sistema de protección social básico universal (seguridad de ingresos y salud básicos).

Para que estas líneas de política puedan ser implementadas es imprescindible que el aparato recaudatorio se robustezca y se profundice. Por ello, es clave el estudio de políticas de financiamiento que permitan el desarrollo sostenible tanto a nivel de recaudación y de gasto, y considerando los impactos tanto directos como indirectos de estas políticas.

De acuerdo con OECD (2011), diversos instrumentos de política ambiental permitirían controlar los desbalances que genera la actividad económica en los sistemas ambientales. En particular, si estos se enfocan en controlar los riesgos del cambio climático y de la pérdida de biodiversidad. Estos instrumentos se tienen que enfocar también en mejorar la gestión de los recursos naturales y de los ecosistemas, con consecuentes ganancias en el bienestar. También deben enfocarse en el diseño de estrategias coherentes que permitan eliminar la incertidumbre que inhibe la inversión y la innovación que retrasan el desarrollo. A continuación se plantean una serie de políticas que podrían ser aplicadas con el fin de buscar un desarrollo más inclusivo y ambientalmente sostenible. En particular, deben considerarse como mitigadoras de los efectos ambientales, presentar co-beneficios y permitir un mayor crecimiento y equidad social.

De esta forma, se puede distinguir tres grandes líneas de acción como:

1. Implementar rápidamente actividades intensivas en trabajo. En este sentido se requiere que los trabajos sean no peligrosos y ambientalmente limpios. También pueden enfocarse en políticas de monitoreo y gestión de los recursos naturales; en el área de la eficiencia energética con tecnología de punta; en la expansión del área de biocombustibles y del área de captura de carbono.

2. Aumentar la inversión de capital en los recursos naturales y en el medio ambiente. Las áreas donde se recomienda que vayan destinadas estas inversiones son en sectores de control y prevención de la contaminación, en la generación de energía eléctrica sobre fuentes renovables, en la introducción de nuevas fuentes de bio-energía, en el área de la eficiencia energética, en la infraestructura verde de transporte, en áreas que permitan una mayor resiliencia de los recursos naturales frente al cambio climático. 
3. Otros programas específicos con características verdes. Es aconsejable invertir en programas del tipo "cash for clunkers" (efectivo por chatarra) que permita un mayor grado de reciclaje y penetración de nuevas tecnologías. Medidas que permitan la reducción de la congestión, entre otras.

En términos generales, este bloque de medidas permitiría lograr un acercamiento al desarrollo sostenible. En este sentido, es menester recordar que en Junio del año 2012 se celebrará el vigésimo aniversario de la cumbre de la Tierra de Río de Janeiro. En Río +20 existirá una instancia fundamental en la cual los gobiernos puedan acordar políticas que permitan encontrar un punto de inflexión y lograr que la región se acerque hacia un desarrollo más equitativo en el tiempo. 


\section{REFERENCIAS}

CEPAL (Comisión Económica para América Latina y el Caribe) (2011). Panorama de la Inserción Internacional de América Latina y el Caribe 2010-2011. Santiago de Chile: Naciones Unidas.

. (2010a). El Progreso de América Latina y el Caribe hacia los Objetivos de Desarrollo del Milenio. Desafíos para Lograrlos con Igualdad. Santiago de Chile: Naciones Unidas.

--_---o.-. (2010b). La Economía del Cambio Climático en América Latina y el Caribe: Síntesis 2010. Santiago de Chile: Naciones Unidas.

(2010c). La Hora de la Igualdad: Brechas por Cerrar Caminos por Abrir. Santiago de Chile: Naciones Unidas.

IADB (Inter-American Development Bank) (2005). The Millennium Development Goals in Latin America and the Caribbean: Progress, Priorities and IDB Support for Their Implementation. Washington: Inter-American Development Bank.

FAO (Food and Agriculture Organization of the United Nations) (2007). Capacidad de Pesca y Manejo Pesquero en América Latina y el Caribe. Santiago: FAO.

Meadows, D. H., Meadows, D. L., Randers, J. y Behrens III, W. W. (1972). The Limits to Growth: a Report for the Club of Rome's Project on the Predicament of Mankind. New York: Universe Book.

Ocampo, J. A. y Ros, J. (2011). The Oxford Handbook of Latin American Economics. Oxford: Oxford University Press.

OECD (Organisation for Economic Co-operation and Development) (2011). Towards Green Growth. Paris: OECD Publishing.

PNUMA (Programa de Naciones Unidas para el Medio Ambiente) (2011). Perspectivas del Medio Ambiente: América Latina y el Caribe: GEO ALC 3. Panamá: PNUMA.

Prebisch, R. (1980). Biosphere and Development, CEPAL Review, 12, 69-84.

Sachs, J. (2008). Economía para un Planeta Abarrotado. Madrid: Debate.

Stern, N. (2007). The Economics of Climate Change. Londres: Cambridge University Press.

WCED (World Commission on Environment and Development) (1987). Our Common Future. Oxford: Oxford University Press. 